\title{
Metabolism and function of phenazines in bacteria: impacts on the behavior of bacteria in the environment and biotechnological processes
}

\author{
Leland S. Pierson III • Elizabeth A. Pierson
}

Received: 16 January 2010 /Revised: 11 February 2010 / Accepted: 12 February 2010/Published online: 30 March 2010

(C) The Author(s) 2010. This article is published with open access at Springerlink.com

\begin{abstract}
Phenazines constitute a large group of nitrogencontaining heterocyclic compounds produced by a diverse range of bacteria. Both natural and synthetic phenazine derivatives are studied due their impacts on bacterial interactions and biotechnological processes. Phenazines serve as electron shuttles to alternate terminal acceptors, modify cellular redox states, act as cell signals that regulate patterns of gene expression, contribute to biofilm formation and architecture, and enhance bacterial survival. Phenazines have diverse effects on eukaryotic hosts and host tissues, including the modification of multiple host cellular responses. In plants, phenazines also may influence growth and elicit induced systemic resistance. Here, we discuss emerging evidence that phenazines play multiple roles for the producing organism and contribute to their behavior and ecological fitness.
\end{abstract}

Keywords Phenazine - Secondary metabolite .

Electron shuttling $\cdot$ Antibiotic $\cdot$ Biofilm

\section{Introduction}

Phenazines comprise a large group of nitrogen-containing heterocyclic compounds that differ in their chemical and

\footnotetext{
L. S. Pierson III $(\square)$

Department of Plant Pathology and Microbiology,

Texas A\&M University,

202 Horticultural and Forestry Sciences Building,

College Station, TX 77843-2133, USA

e-mail: plpm-head@ag.tamu.edu

E. A. Pierson

Department of Horticultural Sciences, Texas A\&M University,

120A L.F. Peterson,

College Station, TX 77843-2132, USA
}

physical properties based on the type and position of functional groups present. More than 100 different phenazine structural derivatives have been identified in nature, and over 6,000 compounds that contain phenazine as a central moiety have been synthesized (Mavrodi et al. 2006). Bacteria are the only known source of natural phenazines. However, natural and synthetic phenazines are of significant interest because of their potential impact on bacterial interactions and biotechnological processes.

Phenazines have been researched longer than most other bacterial secondary metabolites, with over 5,000 published reports dating from 1954 reported in the National Center for Biotechnology Information (PubMed). These secondary metabolites are produced by a variety of bacteria, especially pseudomonads, and have been studied intensively because of their broad spectrum antibiotic properties and roles in virulence. From a biotechnological perspective, the continuing interest in phenazines is due largely to their physicochemical properties, including their oxidation-reduction (redox) properties and their bright pigmentation and ability to change color with $\mathrm{pH}$ and redox state. Phenazines continue to be used for many diverse applications, including as electron acceptors and donors, as components of fuel cells, as environmental sensors and biosensors, and as central components of antitumor compounds.

In this mini-review, we discuss emerging evidence that phenazines play multiple roles and contribute to the behavior and ecological fitness of the producing bacterium. For example, phenazines modify cellular redox state, act as electron shuttles altering electron flow patterns, contribute to biofilm formation and architecture, act as cell signals that regulate patterns of gene expression, and contribute to the survival of the producer. In eukaryotic hosts and host tissues, phenazines modify numerous host cellular responses. In plants, phenazines also influence growth and elicit induced 
systemic resistance. Of particular interest in defining their functional impact are the observations that bacterial species may produce different and often multiple phenazine derivatives, that the phenazine derivatives are produced in different proportions, and the question of whether the amount or proportion of each derivative produced change during growth or in response to environmental factors. We also will discuss how the properties of phenazines have and continue to be of interest for biological and biotechnological applications.

\section{Phenazines and phenazine producers}

Many phenazine-producing bacteria are commonly found associated with host organisms (Table 1). Perhaps the most studied phenazine is pyocyanin (5-N-methyl-1-hydroxyphenazine), which is blue when oxidized. It is produced by Pseudomonas aeruginosa, a common soil inhabitant and opportunistic human pathogen. Pyocyanin (PYO) was isolated originally from patient wounds and subsequently demonstrated to be associated with infections caused by $P$. aeruginosa. Its presence is associated with high morbidity and mortality in immuno-compromized patients, such as cystic fibrosis patients (Courtney et al. 2007; Murray et al. 2007). Phenazines produced by fluorescent pseudomonads also are studied extensively for their application in plant disease management. The bias in research toward pseudomonads often leads to the perception that phenazines are produced primarily by this group. However, phenazines are produced by a wide variety of Eubacteria including both Gram-negative and Gram-positive species. Phenazine producers include Nocardia, Sorangium, Brevibacterium, Burkholderia, Erwinia, Pantoea agglomerans, Vibrio, Pelagiobacter (see Mavrodi et al. 2006; Mavrodi et al.
2010; Mentel et al. 2009), and members of the Actinomycetes, especially Streptomyces (Turner and Messenger 1986). Additionally, Methanosarcina, a member of the Archaea, was shown to contain a phenazine derivative (Abken et al. 1998). This microbe is known to utilize acetate, methylamines, and methanol. New phenazine producers continue to be identified, such as Brevibacterium sp. KMD 003 isolated from a marine purple sponge (Choi et al. 2009).

It is increasingly evident that bacteria produce a wide variety of phenazines, and that many bacteria produce multiple phenazine derivatives (Fig. 1). Bioinformatic comparisons of the phenazine biosynthetic genes among several bacteria demonstrate a high degree of conservation of five genes (Mavrodi et al. 2006; Mentel et al. 2009; Gross and Loper 2009). These are considered the 'core' biosynthetic genes as each is required for the synthesis of the basic three-ringed phenazine structure. Recent evidence suggests that these 'core' biosynthetic genes moved among diverse bacterial genera via horizontal transmission (Mavrodi et al. 2010). In most phenazine-producing bacteria, the core biosynthetic genes are flanked by one or more accessory genes that encode different terminal-modifying enzymes that result in the production of additional phenazine derivatives. For example, Pseudomonas chlororaphis 3084 , a root-associated beneficial bacterium produces three phenazines, phenazine-1-carboxylic acid (PCA), 2-hydroxyphenazine-1-carboxylic acid (2OHPCA), and 2-hydroxyphenazine (2OHPZ) (Pierson and Thomashow 1992). This Pseudomonas species is unique in that it contains $p h z O$, a gene that encodes a monooxygenase, located immediately downstream of the core genes (Delaney et al. 2001). The presence of $p h z O$ converts a small amount $(\sim 10 \%)$ of the yellow PCA into the bright orange 2OHPCA. Additionally, a third minor derivative, 2OHPZ, is generated spontane-
Table 1 Examples of phenazine-producing microbes commonly associated with hosts

\footnotetext{
${ }^{\text {a }}$ Callysongia (Choi et al. 2009).

${ }^{\mathrm{b}}$ Formerly Erwinia carotovora subsp atroseptica.

${ }^{\mathrm{c}}$ Arthropod endosymbiont (Gebhardt et al. 2002).
}

\begin{tabular}{|c|c|c|c|c|}
\hline Bacterium & Human & Animal & Plant & Insect \\
\hline Brevibacterium linens & $+($ skin $)$ & & & \\
\hline Brevibacterium sp. KMD 003 & & $+(\text { marine sponge })^{\mathrm{a}}$ & & \\
\hline Burkholderia cepacia & + & + & + & \\
\hline Methanosarcina mazei & + (intestine) & + (rumen) & & \\
\hline Mycobacterium abscessus & $+($ skin, soft tissues $)$ & & & \\
\hline Pantoea agglomerans & + & + & + & \\
\hline Pectobacterium atrosepticum ${ }^{\mathrm{b}}$ & & & + & \\
\hline Pelagio variabilis & & + (macroalgae $)$ & & \\
\hline Pseudomonas aeruginosa & + & + & + & + \\
\hline Pseudomonas chlororaphis & & & + & \\
\hline Pseudomonas fluorescens & & & + & \\
\hline Streptomyces anulatus & & & & $+^{\mathrm{c}}$ \\
\hline Streptomyces cinnamonensis & & & + & \\
\hline
\end{tabular}


Fig. 1 Representative phenazine structural derivatives. Phenazine methosulfate and neutral red are commonly used phenazine derivatives. Phenazine-1-carboxylic acid, 2-hydroxy-phenazine-1carboxylic acid and 2-hydroxyphenazine are produced by $P$. chlororaphis strain 30-84. Pyocyanin is produced by $P$. aeruginosa. MLN944 is a DNAbinding agent inhibitory to marine and human tumors (Sappal et al. 2004). Methanophenazine is produced by the Archaea Methanosarcina spp. Descriptions are provided in the text
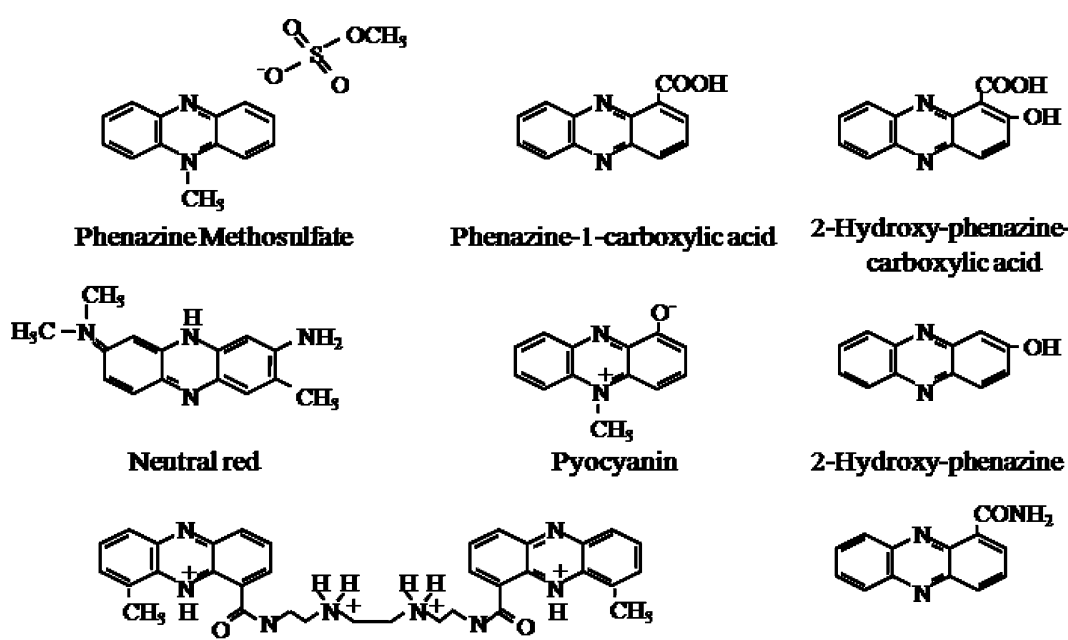

2-Hydroxy-phenazine-1carboxylic acid

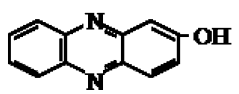

2-Hydroxy-phenarine

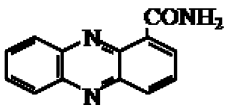

XR5944 anticancer drug

Phenazine-1-carboxamide

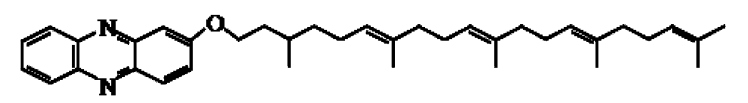

Methanophenazine

ously from 2OHPCA. P. chlororaphis PCL1391 and P. aeruginosa $\mathrm{PAO} 1$ contain $\mathrm{phzH}$, a putative transamidase that converts a portion of PCA into phenazine-1-carboxamide $(\mathrm{PCN})$, enabling these strains to produce both PCA and PCN (Chin-A-Woeng et al. 2001). Two additional genes in $P$. aeruginosa PAO1, $p h z M$, a methyltransferase (Parsons et al. 2007), and $p h z S$, a flavin-containing monooxygenase, together are responsible for the conversion of PCA to pyocyanin (Mavrodi et al. 2001; Greenhagen et al. 2008). $\mathrm{PhzS}$ alone can facilitate conversion of PCA to 1-hydroxyphenazine (1OHPZ). P. aeruginosa PAO1 has two phenazine core biosynthetic gene clusters, separated from each other by ca. 2.6 Mb. One phenazine core region is flanked upstream by $p h z M$ and downstream by $p h z S$. In contrast, the transamidase $p h z H$ is not closely linked to either biosynthetic locus. It is interesting that one or a few terminal modifying enzymes are responsible for the chemical modifications that result in the majority of natural phenazine derivatives. Since these structural modifications account for many of the biological functions of phenazines, the expression of these individual genes greatly affects the ecological fitness and activities of the producing bacterium.

Recent biochemical work has led to important insights into the mechanisms of phenazine biosynthesis, and several excellent reviews are available (Mavrodi et al. 2006; Mavrodi et al. 2010; Gross and Loper 2009). Some phenazine biosynthetic enzymes have been crystallized, and studies of these structures have led to a greater appreciation of the complexities of phenazine synthesis (Mentel et al. 2009; Mavrodi et al. 2010). A brief overview of phenazine biosynthesis is given below. Phenazines are derived from the shikimic acid pathway, using the interme- diate chorismic acid as the branch point for biosynthesis of the basic phenazine aromatic structure (Fig. 2). The shikimic acid biosynthetic pathway is highly conserved and is involved in the production of numerous metabolites necessary for primary growth, including the three aromatic amino acids, and para-aminobenzoic acid. In bacteria, this pathway is under stringent regulation, occurring primarily at the first step involving the condensation of erythrose-4phosphate and phosphoenolpyruvic acid by a type I-3-deoxyD-arabinoheptulosonate-7-phosphate (DAHP) synthase. In many bacteria, isozymes of type I-DAHP synthase exist. Each is subject to feedback inhibition by one of the end products of the pathway. Interestingly, in many phenazine producers, the third gene in the biosynthetic operon $(p h z C)$ encodes a type II-3-DAHP synthase that is more similar to DAHP synthases of solanaceous plants than that of prokaryotes (Pierson et al. 1995). In plants, type II-3-DHAP synthase enzyme lacks a loop region required for allosteric control (Webby et al. 2005). The activity of this enzyme is not modulated by the three aromatic amino acid products, but its activity is enhanced during specific aspects of plant growth such as during seed germination and shoot formation and by glyphosate exposure (Pinto et al. 1988). Expression of $p h z C$ may enhance the first condensation step, ensuring sufficient substrate levels for phenazine production. However, unregulated expression of $p h z C$ may deplete the cell of metabolites required for primary growth and may lead to reduced overall fitness in the rhizosphere (Mavrodi et al. 2006). This need for controlled expression may explain, at least in part, the complex regulation of phenazine production.

Almost all studies on phenazine regulation to date have focused on pseudomonads. These studies show that 
Fig. 2 Phenazine biosynthesis [Figure modified from Mentel et al. (2009)]. Phenazines are derived from the shikimic acid pathway that is highly conserved in most organisms. Chorismic acid serves as the phenazine branch point once the phenazine biosynthetic genes $(p h z A B C D E F G)$ are expressed (note $p h z B, p h z D, p h z E, p h z F$ and $p h z G$ are considered the five core genes). $\mathrm{PhzC}$, the third enzyme in the phenazine operon, is a type II-3-deoxy-D-arabinoheptulosonate-7-phosphate that probably ensures sufficient substrate flow through the shikimic acid pathway for phenazine biosynthesis. The early steps of phenazine biosynthesis are becoming well elucidated while several of the later steps are not yet completely understood and differences may reflect variation in the final biosynthetic steps among microorganisms. From PCA a number of derivatives are formed by additional terminal modifying genes (not shown). For a thorough discussion of the pathway please see Mentel et al. (2009). Abbreviations: DAHP:

3-deoxy-D-arabinoheptulosonate 7-phosphate, Gln: glutamine, Glu: glutamic acid, ADIC: 2amino-2-desoxyisochorismic acid, DHHA: trans-2,3-dihydro3-hydroxyanthranilic acid, PCA: phenazine-1-carboxylic acid

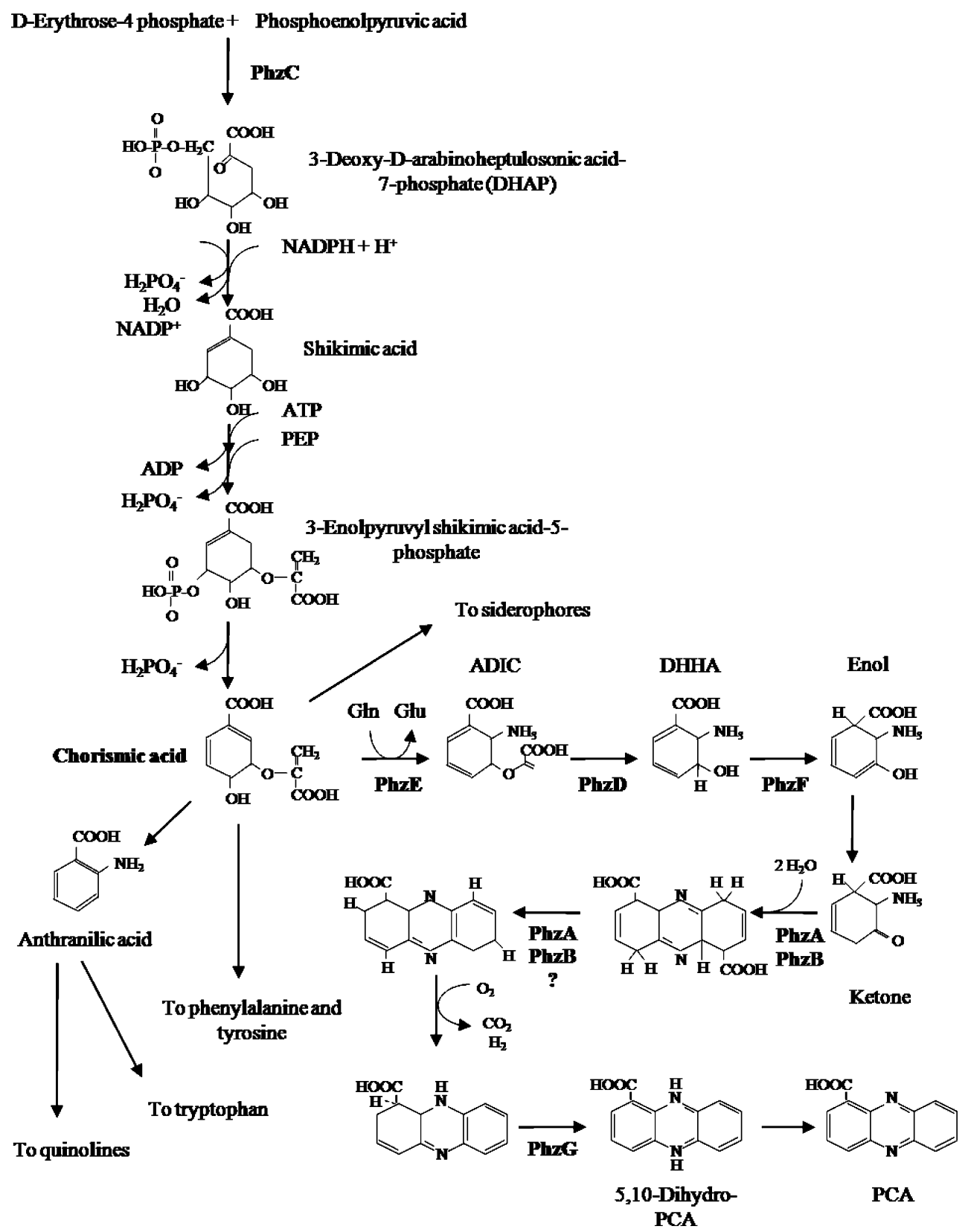

different pseudomonads utilize combinations of conserved regulatory systems integrated into sensory networks to control phenazine production in response to environmental, nutritional, population, and metabolic inputs (reviewed in Mavrodi et al. 2006; Mentel et al. 2009; Gross and Loper 2009). For example, phenazine production by the plantassociated bacteria $P$. chlororaphis strain 30-84 and $P$. fluorescens strain 2-79 is regulated at multiple levels. These include transcriptional regulation via quorum sensing (QS) (PhzR/PhzI), two component positive (GacS/GacA) and two component negative (RpeA/RpeB) regulation. Yet additional regulatory genes have been implicated in $P$. chlororaphis phenazine control, including pip, and post-transcriptional control by $r \operatorname{sm} A$ and $r \sin Z$ (Pierson et al. unpub.).
The opportunistic pathogen $P$. aeruginosa also regulates PYO production by multiple regulatory systems. However, there are distinct differences in the types of regulatory systems and the linkage(s) between regulatory modules. For example, $P$. aeruginosa utilizes the hierarchical LasR/LasI and RhlR/RhlI QS systems to activate PYO production, as well as a third signaling system based on production of 2heptyl-3-hydroxyl-4-quinoline, known as the Pseudomonas quinoline system (PQS) (Dubern and Diggle 2008). $P$. aeruginosa contains the gene $m v f R$ involved in the regulation of the MexGHI-opmD operon believed to be responsible for secretion of the QS signals required for $\mathrm{PYO}$ production. Recently, PA1196 was identified as a regulator of the RhlR/RhlI and PQS systems in that mutation of the 
open reading frame PA1196 resulted in significant decrease in $r h l I$ and $p q s A$ expression as compared to the wild type (Liang et al. 2009). Additional regulatory inputs affecting PYO production are likely to be identified. In contrast to $P$. aeruginosa, hierarchical QS systems and analogs of the PQS system have not been identified in P. chlororaphis or P. fluorescens.

Pseudomonas sp. strain M18, a biological control strain isolated from the melon rhizosphere, has a phenazine biosynthetic locus organization similar to P. aeruginosa, including two separate phenazine core gene clusters and the presence of both the methyltransferase $p h z M$ and the monooxygenase $p h z S$ genes. However, it produces predominantly PCA, whereas $P$. aeruginosa produces primarily PYO. In strain M18, the expression of $p h z M$ and lasI (and a third gene $p t s P$ ) is under temperature-dependent regulation such that they are transcribed at $28^{\circ} \mathrm{C}$ but not at $37^{\circ} \mathrm{C}$ (Huang et al. 2009). In P. aeruginosa strain PAO1, phzM expression is not affected by temperature, perhaps reflecting its need for expression when associated with a human host.

Although much is known about the regulation of the core phenazine biosynthetic genes, especially in Pseudomonas spp., little is known about the fine tuning of the timing of expression and the relative amounts of the different phenazines produced. For example, fine tuning in $P$. aeruginosa occurs by control of QS signals required for PYO production. P. aeruginosa produces two major QS signals, $\mathrm{C}_{4}$-HSL and 3-oxo- $\mathrm{C}_{12}$-HSL, with short and long acyl chains, respectively (reviewed in Williams and Cámara 2009). P. aeruginosa also contains an AHL acylase specific for long acyl chain signals. The presence of this enzyme appears to act as a quorum quencher by turning off the las QS system by degradation of the longer acyl chain signal (Sio et al. 2006). The removal of this QS signal results in loss of PYO production. This acylase, also known as $q s c 112$ or $p v d Q$, is up-regulated during iron deprivation and may repress PYO production during periods of low iron availability. However, it is not produced at high levels at $37^{\circ} \mathrm{C}$, the temperature which $P$. aeruginosa would encounter upon infection of a human host. Although more work is required, this may represent a mechanism by which the bacterium modulates PYO production in response to iron and host availability.

The regulatory complexity governing phenazine production likely reflects the complexity of the roles phenazines play for the producing bacterium (Pierson and Pierson 1996). This observation is consistent with evolutionary theory that metabolically costly metabolites are more likely to be maintained if they serve multiple functions (Wink 2003). Wang et al. (2009) hypothesized that phenazine biosynthesis evolved originally during a period of low oxygen availability, and that some of the effects on bacterial behavior today may not reflect the conditions that drove their early evolution. Evolutionary pressure on bacteria may have selected for different phenazines to serve different roles, and this is reflected by the diversity in the linkage and types of regulatory pathways controlling their expression. Further, since the roles phenazines play for different producers may not be the same, analysis of more than one or two experimental systems may be required to gain a more complete picture of their functions and importance.

\section{Roles in pathogenesis and competition}

Most of the described effects of phenazines during pathogenesis and competition are attributed primarily to their ability to generate reactive oxygen species (ROS) in other organisms and tissues. In some cases, this may result in host beneficial effects, such as the inhibition of pathogenic organisms. In other cases, bacterial virulence is enhanced by phenazine production as it interferes with normal host cell functions. Both of these outcomes are due ultimately to the ability of phenazines to accept or donate electrons because of their aromatic structure. Whether they accept or donate electrons is dependent on their redox potential relative to that of other electron transfer molecules in the cell or in the environment. Early studies on cellular respiratory chains demonstrated the ability of phenazines to uncouple oxidative phosphorylation by shunting electrons from the endogenous pathway in mammalian cells (StewartTull and Armstrong 1971) and in B. subtilis (Bisschop et al. 1979).

Several recent reviews discuss the formation of ROS and oxidative stress by phenazines (Laursen and Nielsen 2004; Mavrodi et al. 2006). For example, PYO production by $P$. aeruginosa plays important roles in pathogenesis during lung infection (Lau et al. 2004a, b; Winstanley and Fothergill 2009). When growing on airway epithelial cells, PYO (and PCA) produced by $P$. aeruginosa can be reduced via the oxidation of glutathione and NADH resulting in increased oxidation levels. The reduced PYO reacts with free oxygen in the lungs, generating ROS. Airway epithelial cells produce lactoperoxidase and related dual oxidases that produce mild oxidants as a defense effective against a number of bacterial intruders, including Staphylococcus aureus, Burkholderia cepacia and P. aeruginosa. However, PYO production by $P$. aeruginosa negates this host defense mechanism by competing with epithelial cell Duox activity for NADPH (Rada and Leto 2009). The generation of these ROS contributes to the virulence of the infection. ROS also may contribute to successful host invasion and disease due to their negative effects on a range of host cell functions, including respiration, ciliary beating, epidermal cell growth, calcium homeostasis, prostaglandin release, neutrophil 
apoptosis, interleukin-2 release, Immunoglobulin G secretion, and a protease-antiprotease activity (Hassan and Fridovich 1980; Ran et al. 2003; Laursen and Nielsen 2004)

PYO production may inhibit the growth of competing microorganisms during mixed lung infections (Machan et al. 2001). In fact, the effects of phenazines can be simultaneously positive and negative within the same host. For example, while PYO production by $P$. aeruginosa during lung infection of cystic fibrosis patients is correlated with a poor prognosis, it is also highly correlated with a lower incidence of yeast infections (Lau et al. 2004a, b; Cogen et al. 2008). However, a more fundamental role for PYO in the producing bacterium is suggested by the observation that PYO production is important for persistence even in the absence of competitors (Hassett et al. 2009; Kobayashi et al. 2009; Price-Whelan et al. 2006). PYO-producing cells protect themselves by the production of high levels of superoxide dismutase activity (SOD). Mutants of $P$. aeruginosa in $\operatorname{sod} A$ or $\operatorname{sod} B$ are defective in SOD production and produced half or no PYO, respectively, as compared to the wild type (Hassett et al. 1995). Recent work with $P$. aeruginosa and Bacillus subtilis further supports the role of ROS in growth inhibition and bacterial competition. B. subtilis cells containing intact nos genes that produce nitric oxide (NO) that stimulates SOD expression were more resistant to PYO in competition studies compared to isogenic nos mutants (Gusarov et al. 2009). Although the multiple roles of PYO in virulence are being recognized, it should be noted that in addition to PYO, $P$. aeruginosa produces several other phenazine derivatives including 1-hydroxyphenazine, PCA, PCN, aeruginosin A, and aeruginosin B (see Mavrodi et al. 2006) for which the impacts on virulence are less well understood.

In addition to their involvement in pathogenesis and virulence, interest in phenazines as beneficial compounds emerged in the 1980s due to their effectiveness as antimicrobial compounds in plant disease control. Phenazine production by a number of soil-borne bacteria was shown to control a wide range of plant pathogenic fungi (Chin-AWoeng et al. 2003; Mavrodi et al. 2006). For example, $P$. chlororaphis strain 30-84 produces three $\mathrm{PZ}$ derivatives PCA, 2OHPCA, and 2OHPZ (Pierson and Thomashow 1992). P. fluorescens strain 2-79 produces only PCA (Thomashow and Weller 1988), whereas P. chlororaphis strain PCL1391 produces phenazine-1-carboxamide (PCN) and some PCA (Chin-A-Woeng et al. 1998). PCN was shown to control Fusarium oxysporum f. sp. radicis lycopersici, the causative agent of tomato foot and root rot. Both PCA and PCN are involved in control of Pythium myriotylum, the causative agent of root rot of cocoyam (Tambong and Hofte 2001) whereas PYO produced by $P$. aeruginosa inhibited Septoria tritici of wheat (Flaishman et al. 1990). PCA and PCN produced by Pseudomonas strain PCL1391 induced several ABC transporters in Botrytis cinerea (Schoonbeek et al. 2002). Additionally, the ability to produce phenazine was strongly correlated with bacterial persistence in natural soil in the presence of the indigenous microbial community (Mazzola et al. 1992).

These results are consistent with the commonly held belief that the primary role of phenazines produced by these soil-borne bacteria is as antibiotic compounds that aid in their competitive survival in natural systems. Yet, despite the observations that phenazines produced by biological control agents do inhibit a broad spectrum of target fungal pathogens, in many cases, the same phenazine derivatives are ineffective at inhibiting the growth of co-occurring bacteria, probably their most immediate competitors (Fernando and Pierson, unpub.; Beifuss and Tietze 2005). Within rhizosphere communities, the effects of phenazine production on the indigenous rhizosphere community are negligible, with little effect on the microorganisms that compete with the producer (Mavrodi et al. 2006: Dwivedi et al. 2009). More recent studies on phenazine function suggest that, although phenazines may assist the producing bacterium in competitive survival, this is probably not their primary function. As described below, phenazines also induce plant defense pathways, play roles in electron shuttling, iron chelation, biofilm formation, and even serve as signals that modulate gene expression (Fig. 3).

\section{Induction of plant defense pathways}

Phenazines have been shown to activate induced systemic resistance (ISR) in plants. ISR is widespread and can be induced by a number of bacterial components, including outer membrane components, lipopolysaccharide, flagella, siderophores, and volatile compounds (Van Wees et al. 2008). ISR is dependent on expression of the plant ethylene and jasmonic acid pathways, leading to a rapid systemic expression of broad spectrum resistance against numerous pathogens (Verhagen et al. 2004). Inoculation of $P$. aeruginosa onto rice plants elicited ISR, as evidenced by resistance to the fungal rice blast pathogen Magneporthe grisea. PYO was shown to be critical for this induction as loss of PYO production resulted in the loss of ISR against M. grisea (De Vleesschauwer et al. 2006). However, the production of PYO resulted in increased susceptibility of rice to another fungal pathogen, Rhizoctonia solani.

\section{Electron shuttling and iron chelation}

Microorganisms often experience environments in which terminal electron acceptors are limited or may become 
Fig. 3 Representative effects of phenazines on cellular physiology, gene expression, host functions and biotechnological applications. In the center is the basic phenazine structure lacking any additional modifications. Details of the effects of phenazine derivatives on specific functions are described in the text

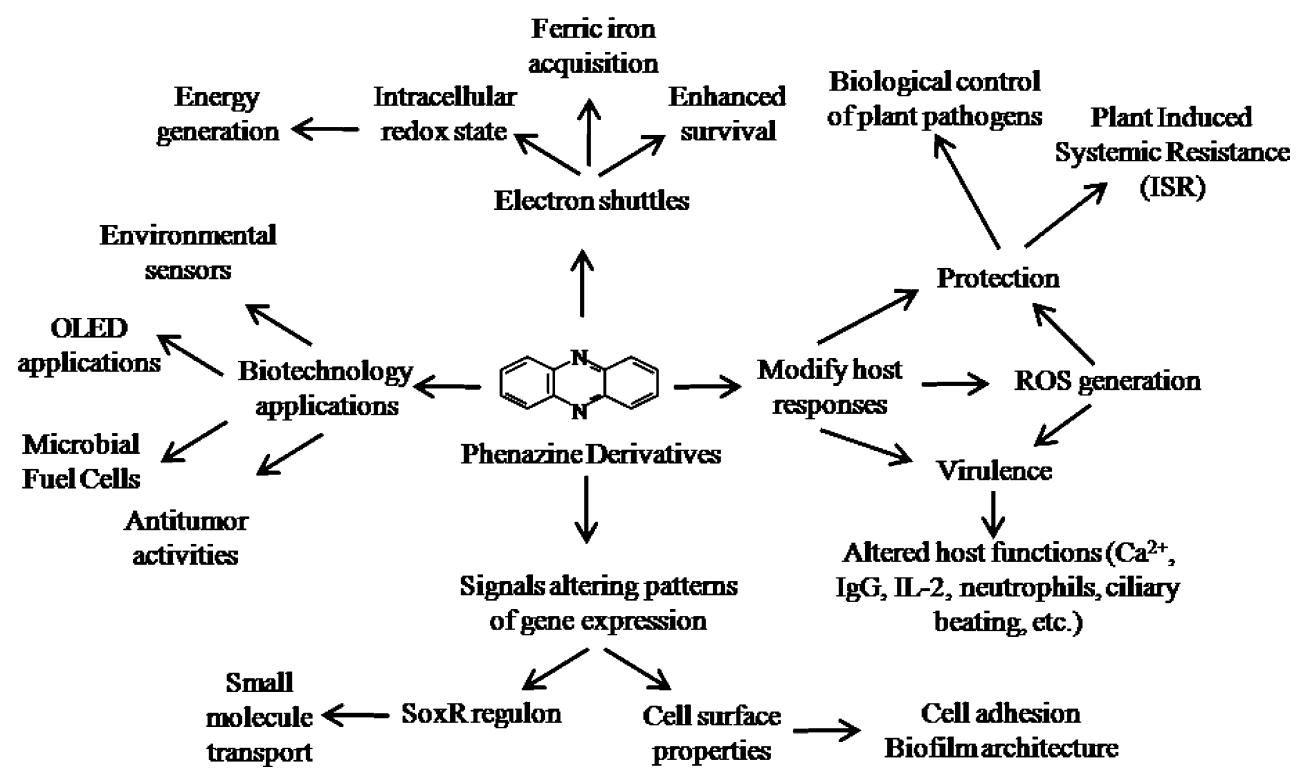

limited under specific conditions. The lack of available terminal electron acceptors severely limits bacterial growth and survival due to low energy generation. In most bacteria, this also results in a highly reduced intracellular redox state as indicated by a high NADH/NAD ${ }^{+}$ratio (de Graef et al. 1999). P. aeruginosa is capable of maintaining a NADH/ $\mathrm{NAD}^{+}$ratio slightly greater than one due to the production of PYO, which serves as an alternate electron acceptor that reoxidizes $\mathrm{NADH}$ to $\mathrm{NAD}^{+}$. This enables the cell to balance intracellular redox in the absence of other electron acceptors (Price-Whelan et al. 2007). Recently, PYO production was shown to be important for bacterial survival, but not growth, under anaerobic conditions (Wang et al. 2009). In this work, wild type $P$. aeruginosa PA14 and a phenazine null derivative were grown as planktonic cultures to stationary phase and electron flow measured in anaerobic bioreactors. The pyocyanin-producing wild type strain remained viable for 7 days, although its population did not increase from its initial level. In contrast, the nonpyocyanin-producing mutant population decreased logarithmically after 3 days. This effect was specific to pyocyanin, as several other redox compounds with similar structures did not support survival. These results demonstrate that phenazines are essential for long term survival under anaerobic conditions, for example, as occurs below the outer surface layer of biofilms (Drago 2009).

In soils, water saturation and microbial and root respiration limit available $\mathrm{O}_{2}$ as a terminal electron acceptor. The use of alternative electron acceptors such as ferric $\left(\mathrm{Fe}^{3+}\right)$ iron allows continued energy generation and enhanced microbial survival. The root-associated bacterium P. chlororaphis PCL1391 utilizes phenazine-1-carboxamide (PCN) to efficiently convert ferric $\mathrm{Fe}^{3+}$ hydroxides to ferrous $\mathrm{Fe}^{2+}$ under acidic conditions. The dissimilatory iron-reducing bacterium Shewanella oneidensis MR1 also is capable of utilizing exogenously added PCN to mineralize poorly crystalline $\mathrm{Fe}^{3+}$ hydroxides, enabling it to grow under conditions with limited electron acceptors (Hernandez et al. 2004). Different phenazine structural derivatives have different reactivities to ferrihydrite and hematite $(\mathrm{pH} 5-8)$, suggesting that particular phenazines may react preferentially with specific forms of iron (Wang and Newman 2008). The recent finding that addition of strong iron chelators inhibited $P$. aeruginosa biofilms, especially under anaerobic conditions (O'May et al. 2009), reinforces the probable role of phenazines as electron shuttles to alternative electron acceptors.

One benefit of phenazine-mediated iron reduction is the increased bioavailability of this limited element to the bacterium. For example, PYO produced by $P$. aeruginosa can acquire iron from the human iron chelator transferrin, and the highest rate of iron acquisition occurs under low $\mathrm{O}_{2}$ conditions (Cox 1986). In soils, PYO production may increase iron availability to other organisms. PYO itself was shown recently to act as an iron chelator (Newman unpub.).

The use of phenazines as electron shuttles is not limited to Eubacteria. Methanosarcina spp. generate methane using anaerobic respiration. These Archaea contain a unique energy-saving electron transport system that utilizes methanophenazine as a membrane-localized component. This chain is proposed to consist of two systems: reduced coenzyme $\mathrm{F}_{420}\left(\mathrm{~F}_{420} \mathrm{H}_{2}\right.$ : heterodisulfide oxidoreductase $)$ and $\mathrm{H}_{2}$ : heterodisulfide oxidoreductase. The net result of either system is the sequential transfer of electrons from $\mathrm{F}_{420} \mathrm{H}_{2}$ or $\mathrm{H}_{2}$ to the membrane-localized methanophenazine (MP) with the concurrent release of two protons outside the cell membrane. The reduced methanophenazine $\left(\mathrm{MPH}_{2}\right)$ subsequently donates electrons via the enzyme heterodisulfide reductase (Hdr) to the heterodisulfide of coenzyme $M$ and 
coenzyme B (CoM-S-S-CoB), which serves as the terminal electron acceptor and is involved subsequently in methane production. The net result of each system is the translocation of four protons outside the cell for each pair of electrons. The proton motive force generated is used for ATP synthesis (Abken et al. 1998; Beifuss and Tietze 2005; Kulkarni et al. 2009).

\section{Cell adhesion, biofilm development and dispersal}

Biofilm formation is important for the persistence and survival of bacteria (Davies 2002; Balasubramanian and Mathee 2009; McBain 2009). Many Gram-negative bacteria require functional QS systems in order to form biofilms (Mavrodi et al. 2006; De Sordi and Mühlschlegel 2009; Dobretsov et al. 2009). Recent work with P. chlororaphis 30-84 reinforced the importance of QS in biofilm formation (Maddula et al. 2006). This work also demonstrated that it was specifically the production of phenazines controlled by the PhzR/I QS that was critical. Mutants defective in the $\mathrm{PhzR} / \mathrm{I}$ QS system failed to establish biofilms even after 6 days, similar to a phenazine structural mutant. Introduction of functional copies of $p h z R / p h z I$ in trans failed to rescue the phenazine structural mutant for adhesion and biofilm development, whereas constitutive phenazine expression resulted in earlier biofilm formation after 1-3 days.

P. chlororaphis strain $30-84$ produces primarily phenazine1-carboxylic acid (PCA) and only about $10 \%$ is converted into 2-hydroxy-phenazine-1-carboxylic acid (2OHPCA). Therefore, the effect of altering the ratio of PCA to 2OHPCA produced by strain 30-84 on biofilm development was examined (Maddula et al. 2008). A derivative in which $p h z O$ was inactivated was constructed along with a derivative in which additional copies of $p h z O$ were introduced in trans, resulting in strains that produced only PCA or increased levels of 2OHPCA. Compared to wild type strain 30-84 or the PCA-only producer, the 2OHPCA overproducer adhered more quickly and more uniformly to glass surfaces ( $44 \%$ total coverage as compared to $1 \%$ by the wild type or PCA-only strain after $45 \mathrm{~min}$.). Additionally, the 2OHPCA overproducer formed thicker biofilms than the wild type, but had a cell bio-volume similar to the wild type. In contrast, the PCA-only producer had a thicker biofilm with four-fold higher bio-volume of cells than either the wild type or 2OHPCA overproducer. Dispersion from the biofilm also was reduced in the 2OHPCA overproducer. These results suggest that 2OHPCA may facilitate cellular adhesion, whereas PCA may facilitate growth within the biofilm, possibly as an electron shuttle within the microaerophilic community. The idea that the phenazines produced by $P$. chlororaphis 30-84 may play different roles in biofilm structure and function is intriguing given that early work showed that 2OHPCA had a greater ability to inhibit the growth of some microorganisms than PCA (Toohey et al. 1965). Moreover, P. chlororaphis 30-84 was found to be more effective against the fungal plant pathogen Gaeumannomyces graminis var. tritici than strains that produce PCA alone. These results suggest that bacteria may produce different phenazine structural derivative in specific concentrations due to the diverse roles they serve for the population.

\section{Phenazines as signals}

The observation that alterations in the levels of specific phenazine structures had significant impacts on cell adhesion and biofilm architecture raises an interesting question as to the mechanism(s) involved in these effects. One possibility is that phenazines, regulated by QS signals, are themselves signals capable of altering patterns of gene expression. Recently, experimental support for this hypothesis was established using $P$. aeruginosa PAO1 (Dietrich et al. 2006). A mutant was constructed in which both sets of phenazine biosynthetic genes were deleted. Transcriptome analysis of the mutant strain, grown with or without $0.2 \mathrm{mM}$ purified PYO, revealed altered expression of 51 genes. Of these, 8 of the 22 genes up-regulated by PYO addition were efflux transporters, while 7 of the 29 genes down-regulated were involved in ferric iron uptake. This work demonstrated that PYO was itself a signal that modulated the physiology of $P$. aeruginosa.

The effects of PYO were shown subsequently to be dependent on two mechanisms, one involving a homologue of E. coli SoxR and a second dependent on another unknown regulator (Kobayashi and Tagawa 2004). In E. coli, SoxR regulates the expression of superoxide stress response regulons in conjunction with a second regulator, SoxS. Dietrich et al. (2008) used bioinformatic and gene expression analysis to study the role of SoxR regulons in $P$. aeruginosa and Streptomyces coelicolor. In P. aeruginosa and $S$. coelicolor, many of the SoxR regulons identified lacked genes involved in superoxide stress response, but contained genes involved in the transport of small molecules. One of the products under SoxR control in $P$. aeruginosa PA14 is the MexGHI-OpmD efflux system involved in transport of PCA and a red phenazine. Interestingly, PYO stimulated the expression of this efflux system, although it was not dependent on it for release from the cell. Comparison of wild type PA14, a soxR deletion mutant, and a PYO-overproducing strain indicated that the presence of higher levels of PYO resulted in the maintenance of a smooth colony morphology as compared to the wild type PA14. The higher levels of PYO in the soxR deletion and PYO-overproducing mutants is presumably 
due to the inability of the soxR mutant to transport phenazines out of the cell and the overproducing strain having more phenazine produced as compared to the wild type. These results suggested that the diffusibility of phenazines may influence colony phenotype over distance.

In $P$. chlororaphis, transcriptome analysis was used to compare a phenazine structural mutant to the wild type strain. In this experiment, phenazine production occurred naturally under QS regulation. In preliminary experiments, $63(1.1 \%)$ and $41(0.8 \%)$ P. chlororaphis genes were up- or down-regulated by phenazine production, respectively (Pierson et al. unpub). In contrast to $P$. aeruginosa, many of the genes that appeared to be activated encode products associated with cell adhesion and biofilm development, including fimbrial and LPS genes. Although it is clear that phenazines can serve as signals in both Pseudomonas species, the effects of this signaling may not be the same for each species. Further, in cases where multiple phenazines are produced, distinct phenazines may effect the expression of different or overlapping gene sets. These differences may be useful tools in understanding the evolutionary selection experienced by each species.

\section{Biotechnological applications}

The ability of phenazines to promote electron transfer has many realized and potential biotechnological applications. Phenazines long have been used as colorimetric redox indicators. The $\mathrm{pH}$ indicator neutral red is among the best known. More recently, phenazines have been utilized for the development of sensors and in nanotechnology. For example, a phenazine derivative was used to develop a luminescence-based pH sensor (Ryazanova et al. 2007) and an amperometric sensor for hydrogen peroxide determination utilizing neutral red attached to multiwalled carbon nanotubes was developed (Jeykumari and Narayanan 2007).

Microbial fuel cells (MFC) use microorganisms to catalyze the conversion of chemical energy into electrical energy (Torres et al. 2010). An ongoing issue with MFCs is that the slow rate of electron transfer from the microorganism to the anodic electrode limits MFC efficiency. Early work demonstrated that phenazine methosulfate or phenazine ethosulfate served as good electron acceptors in photoelectrochemical cells (Sanderson et al. 1987). More recently, it was observed that other phenazines also contributed to the rates of electron transfer in MFCs. The addition of pyocyanin to MFC-containing Brevibacillus sp. PTH1 doubled the rate of electron transfer (Rabaey et al. 2005). The addition of a PCA-producing P. chlororaphis or a derivative that produces high levels of $\mathrm{PCN}$ to a mixed MFC also resulted in higher electron transfer rates (Pham et al. 2008). A strain of $P$. aeruginosa was isolated from MFC maintained in a batch mode for over a year that supported $352 \mathrm{mV}$ using $1500 \mathrm{mg} / \mathrm{l}$ glucose as fuel (Luo et al. 2009).

Phenazines conjugated to other compounds offer potential as components of organic light emitting devices (OLED), such as a phenanthroline-fused phenazine (Chen and Xiao-Chang 2004). OLEDs are gaining popularity due to their low voltage requirements, wide color range, and light weight. OLEDs are organic semiconductors containing an emissive layer placed between a transparent anode (e.g., transparent indium tin oxide) and a metal cathode (e.g., $\mathrm{Mg}$, $\mathrm{Al}, \mathrm{Ag})$. When a bias is applied across the electrodes, the 'holes' (areas lacking electrons) and electrons combine in the emissive layer resulting in light emission ( $\mathrm{Li}$ et al. 2009).

Phenazines are associated with antitumor activities (Laursen and Nielsen 2004; Mavrodi et al. 2006). Cells that are actively respiring, such as tumor cells, appear to be more susceptible to respiratory interference and ROS generation caused by phenazine compounds. Additionally, phenazine derivatives known to interfere with topoisomerase I and II activities in eukaryotic cells have been identified. Cancer cells, having high levels of both topoisomerases, are more susceptible to this interference. For example, active proliferation of human lymphocytes was inhibited by pyocyanin (Sorensen et al. 1983). The development of synthetic anticancer phenazine derivatives is an ongoing area of research aimed at combining known phenazine biological activities with increased target specificity towards cancer cells (Nakaike et al. 2005; Hari et al. 2009).

\section{Concluding remarks}

The roles of phenazines in biotechnology appear limitless based on their ability to shuttle electrons and new applications based on other properties are likely to be identified in the future. In natural systems, phenazineproducing bacteria often produce multiple derivatives and even individual phenazines appear to play multiple roles in bacterial interactions and behaviors. Future research needs to address the importance of the relative quantities of phenazine derivatives produced and how this balance is maintained. For example, how does changing the amount of one phenazine derivative produced relative to another affect bacterial fitness? Understanding the patterns of gene expression regulating the timing and levels of phenazines produced may lead to greater appreciation of the reasons bacteria produce diverse phenazine structural derivatives at different levels and perhaps a better understanding of the complex roles these phenazines serve in the lifestyles and behavior of bacteria. 


\section{Summary}

- Phenazines constitute a large group of nitrogencontaining heterocyclic compounds that differ in their chemical and physical properties based on the type and position of the functional groups present.

- Most studies on phenazines have focused on Gramnegative bacteria such as Pseudomonas spp. The recognition that phenazines are produced by a wide variety of Eubacteria, and some Archaea, indicates a need for research on the regulation and functions of phenazines in other classes of bacteria.

- Many phenazine-producing bacteria commonly form associations with different hosts, although this may reflect a bias in how phenazine producing bacteria have been selected.

- Phenazine derivatives with chemical modifications at one or more positions of the aromatic ring structures have been identified, and most are due to one or a few terminal modifying enzymes. These differences in phenazine structure impact their biological functions, and therefore these modifications may determine the ecological niche the bacterium occupies.

- Phenazine production is controlled by regulatory networks that are organized differently in each species. Although phenazines serve multiple roles for the producing organism, the roles phenazines play for each producers may not be the same. Therefore, conclusions based on one or two experimental systems may provide only partial insights into their importance.

- Phenazines serve as signals, altering patterns of gene expression in the producing bacterium. However, the specific genes may be different in each bacterial species. Future work will determine if different phenazine structural derivatives affect the expression of a conserved or overlapping groups of genes, and how these genes affect bacterial behaviors.

- In order to gain a more complete understanding of the impacts of phenazines on bacterial behavior and fitness, future studies should address:

- why strains produce specific and often more than one phenazine derivative,

- why these derivatives are produced in distinct quantities or ratios, and

- how these ratios are "fine-tuned."

- Based on the knowledge gained from these studies, future biotechnological advances may come from the use of finely-tuned ratios of phenazines rather than relying on single derivatives.

Acknowledgements The authors wish to thank Dr. Dianne Newman for unpublished results. We also thank Dr. Martha Hawes and Dr. Anne Estes for their review comments. Parts of this work were supported by NSF Grant No. 0545804 and by USDA-NRI Grant No. 2008-00657.
Open Access This article is distributed under the terms of the Creative Commons Attribution Noncommercial License which permits any noncommercial use, distribution, and reproduction in any medium, provided the original author(s) and source are credited.

\section{References}

Abken HJ, Tietze M, Brodersen J, Bäumer S, Beifuss U, Deppenmeier U (1998) Isolation and characterization of methanophenazine and function of phenazines in membrane- bound electron transport of Methanosarcina mazei Gö1. J Bacteriol 180:2027-2032

Balasubramanian D, Mathee K (2009) Comparative transcriptome analyses of Pseudomonas aeruginosa. Hum Genomics 3:349-361

Beifuss U, Tietze M (2005) Methanophenzine and other natural biologically active phenazines. Top Curr Chem 244:77-113

Bisschop A, Bergsma J, Konings WN (1979) Site of interaction between phenazine methosulphate and the respiratory chain of Bacillus subtilis. European Journal of Biochemistry 93:369-374

Chen JP, Xiao-Chang CL (2004) Organic light-emitting device having phenanthroline-fused phenazine. US Patent 6713781

Chin-A-Woeng TFC, Bloemberg GV, Lugtenberg BJJ (2003) Phenazines and their role in biocontrol by Pseudomonas bacteria. New Phytologist 157:503-523

Chin-A-Woeng TFC, Bloemberg GV, van der Bij AJ, van der Drift KMGF, Schripsema J, Kroon B, Scheffer RJ, Keel C, Bakker PAHM, Tichy HV, de Bruijn FJ, Thomas-Oates JE, Lugtenberg BJJ (1998) Biocontrol by phenazine-1-carboxamide-producing Pseudomonas chlororaphis PCL1391 of tomato root rot caused by Fusarium oxysporum f sp radicis-lycopersici. Mol PlantMicrob Interact 11:1069-1077

Chin-A-Woeng TFC, Thomas-Oates JE, Lugtenberg BJJ, Bloemberg GV (2001) Introduction of the $p h z H$ gene of Pseudomonas chlororaphis PCL1391 extends the range of biocontrol ability of phenazine-1-carboxylic acid-producing Pseudomonas spp strains. Molecular Plant-Microbe Interactions 14:1006-1015

Choi EJ, Kwon HC, Ham J, Yang HO (2009) 6-Hydroxymethyl-1phenazine-carboxamide and 1, 6-phenazinedimethanol from a marine bacterium, Brevibacterium sp KMD 003, associated with marine purple vase sponge. J Antibiot 62:621-624

Cogen AL, Nizet V, Gallo RL (2008) Skin microbiota: a source of disease of defence? British Journal of Dermatology 158:442-455

Courtney JM, Bradley J, McCaughan J, O'Connor TM, Shortt C, Bredin CP, Bradbury I, Elborn JS (2007) Predictors of mortality in adults with cystic fibrosis. Pediatric Pulmonology 42:525-532

Cox C (1986) Role of pyocyanin in the acquisition of iron from transferrin. Infection and Immunity 52:263-270

Davies JC (2002) Pseudomonas aeruginosa in cystic fibrosis: pathogenesis and persistence. Paediatr Respir Rev 3:128-134

de Graef MR, Alexeeva S, Snoep JL, Teixeira de Mattos MJ (1999) The steady-state internal redox state (NADH/NAD) reflects the external redox state and is correlated with catabolic adaptation in Escherichia coli. J Bacteriol 181:2351-2357

Delaney SM, Mavrodi DV, Bonsall RF, Thomashow LS (2001) phzO, a gene for biosynthesis of 2-hydroxylated phenazine compounds in Pseudomonas aureofaciens 30-84. J Bacteriol 183:318-327

De Sordi L, Mühlschlegel FA (2009) Quorum sensing and fungalbacterial interactions in Candida albicans: a communicative network regulating microbial coexistence and virulence. FEMS Yeast Res 9:990-999

Dietrich LE, Price-Whelan A, Petersen A, Whiteley M, Newman DK (2006) The phenazine pyocyanin is a terminal signaling factor in the quorum sensing network of Pseudomonas aeruginosa. Molecular Microbiology 61:1308-1321 
Dietrich LE, Teal TK, Price-Whelan A, Newman DK (2008) Redoxactive antibiotics control gene expression and community behavior in divergent bacteria. Science 321:1203-1206

Dobretsov S, Teplitski M, Paul V (2009) Mini-review: quorum sensing in the marine environment and its relationship to biofouling. Biofouling 25:413-427

De Vleesschauwer D, Cornelis P, Höfte M (2006) Redox-active pyocyanin secreted by Pseudomonas aeruginosa 7NSK2 triggers systemic resistance to Magnaporthe grisea but enhances Rhizoctonia solani susceptibility in rice. Molecular Plant-Microbe Interactions 19:1406-1419

Drago L (2009) Bacteria and biofilm in respiratory tract infections. Infect Med 17(Suppl 2):3-9

Dubern JF, Diggle SP (2008) Quorum sensing by 2-alkyl-4-quinolones in Pseudomonas aeruginosa and other bacterial species. Mol Biosyst 4:882-888

Dwivedi D, Johri BN, Ineichen K, Wray V, Wiemken A (2009) Impact of antifungals producing rhizobacteria on the performance of Vigna radiate in the presence of arbuscular mycorrhizal fungi. Mycorrhiza 19:559-570

Flaishman M, Eyal Z, Voisard C, Haas D (1990) Suppression of Septoria tritici by Phenazine- or Siderophore-deficient mutants of Pseudomonas. Curr Microbiol 20:121-124

Gebhardt K, Schimana J, Krastel P, Dettner K, Rheinheimer J, Zeeck A, Fiedler HP (2002) Endophenazines A-D, new phenazine antibiotics from the arthropod associated endosymbiont Streptomyces anulatus: I. Taxonomy, fermentation, isolation and biological activities. J Antibiot (Tokyo) 55:794-800

Greenhagen BT, Shi K, Robinson H, Gamage S, Bera AK, Ladner JE, Parsons JF (2008) Crystal structure of the pyocyanin biosynthetic protein PhzS. Biochemistry 47:5281-5289

Gross H, Loper JE (2009) Genomics of secondary metabolite production by Pseudomonas spp. Natural Product Reports 26:1408-1446

Gusarov I, Shatalin K, Staroodubtseva M, Nudler E (2009) Endogenous nitric oxide protects bacteria against a wide spectrum of antibiotics. Science 325:1380-1384

Hari N, Moorthy NS, Karthikeyan C, Trivedi P (2009) Synthesis, cytotoxic evaluation and in silico pharmacokinetic prediction of some benzo $[\alpha]$ phenazine-5-sulfonic acid derivatives. Med Chem 5:549-557

Hassan HM, Fridovich I (1980) Mechanism of action of pyocyanin. J Bacteriol 141:156-163

Hassett DJ, Schweizer HP, Ohman DE (1995) Pseudomonas aeruginosa $\operatorname{sod} A$ and $\operatorname{sod} B$ mutants defective in manganese- and ironcofactored superoxide dismutase activity demonstrate the importance of the iron-cofactored form in aerobic metabolism. J Bacteriol 177:6330-6337

Hassett DJ, Sutton MD, Schurr MJ, Herr AB, Caldwell CC, Matu JO (2009) Pseudomonas aeruginosa hypoxic or anaerobic biofilm infections within cystic fibrosis airways. Trends in Microbiology 17:130-138

Hernandez ME, Kappler A, Newman DK (2004) Phenazines and other redox-active antibiotics promote microbial mineral reduction. Applied and Environmental Microbiology 70:921-928

Huang J, Xu Y, Zhang H, Li Y, Huang X, Ren B, Zhang X (2009) Temperature-dependent expression of $p h z M$ and its regulatory genes lasI and ptsP in rhizosphere isolate Pseudomonas sp strain M18. Applied and Environmental Microbiology 75:6568-6580

Jeykumari DRS, Narayanan SS (2007) Covalent modification of multiwalled carbon nanotubes with neutral red for the fabrication of an amperometric hydrogen peroxide sensor. Nanotechnology 18:125501-125510

Kobayashi H, Kobayashi O, Kawai S (2009) Pathogenesis and clinical manifestations of chronic colonization by Pseudomonas aeruginosa and its biofilms in the airway tract. J Infect Chemother 15:125-142
Kobayashi K, Tagawa S (2004) Activation of SoxR-dependent transcription in Pseudomonas aeruginosa. J Biochem 136:607615

Kulkarni G, Kridelbaugh DM, Guss AM, Metcalf WW (2009) Hydrogen is a preferred intermediate in the energy-conserving electron transport chain of Methanosarcina barkeri. Proceedings of the National Academy of Sciences of the United States of America 106:15915-15920

Lau GW, Hassett DJ, Ran H, Kong F (2004a) The role of pyocyanin in Pseudomonas aeruginosa infection. Trends Mol Med 10:599606

Lau GW, Ran H, Kong F, Hassett DJ, Mavrodi D (2004b) Pseudomonas aeruginosa pyocyanin is critical for lung infection in mice. Infection and Immunity 72:4275-4278

Laursen JB, Nielsen J (2004) Phenazine natural products: Biosynthesis, synthetic analogues, and biological activity. Chemical Reviews 104:1663-1685

Li XN, Wu ZJ, Si ZJ, Liang-Zhou LXJ, Zhang HJ (2009) Effect of secondary ligands' size on energy transfer and electroluminescent efficiencies for a series of europium(III) complexes, a density functional theory study. Phys Chem Chem Phys 11:9687-9695

Liang H, Li L, Kong W, Shen L, Duan K (2009) Identification of a novel regulator of the quorum-sensing systems in Pseudomonas aeruginosa. FEMS Microbiology Letters 293:196-204

Luo HP, Liu GL, Zhang RD, Cao LX (2009) Isolation and characterization of electrochemical active Pseudomonas aeruginosa strain RE7. Huanjing Kexue 30:2118-2123

Machan ZA, Pitt TL, White W, Watson D, Taylor GW, Cole PJ, Wilson R (2001) Interaction between Pseudomonas aeruginosa and Staphylococcus aureus: description of an anti-staphylococcal substance. J Med Microbiol 34:213-217

Maddula VS, Pierson EA, Pierson LS 3rd (2008) Altering the ratio of phenazines in Pseudomonas chlororaphis (aureofaciens) strain 30-84: effects on biofilm formation and pathogen inhibition. $\mathrm{J}$ Bacteriol 190:2759-2766

Maddula VS, Zhang Z, Pierson EA, Pierson LS 3rd (2006) Quorum sensing and phenazines are involved in biofilm formation by Pseudomonas chlororaphis (aureofaciens) strain 30-84. Microbial Ecology 52:289-301

Mavrodi DV, Blankenfeldt W, Thomashow LS, Mentel M (2006) Phenazine compounds in fluorescent Pseudomonas spp biosynthesis and regulation. Annual review of Phytopathology 44:417445

Mavrodi DV, Bonsall RF, Delaney SM, Soule MJ, Phillips G, Thomashow LS (2001) Functional analysis of genes for biosynthesis of pyocyanin and phenazine-1-carboxamide from Pseudomonas aeruginosa PAO1. J Bacteriol 183:6454-6465

Mavrodi DV, Peever TL, Mavrodi OV, Parejko JA, Raaijmakers JM, Lemanceau P, Mazurier S, Heide L, Blankenfeldt W, Weller DM, Thomashow LS (2010) Diversity and Evolution of the Phenazine Biosynthesis Pathway Appl Environ Microbiol [Epub ahead of print]

Mazzola M, Cook RJ, Thomashow LS, Weller DM, Pierson LS 3rd (1992) Contribution of phenazine antibiotic biosynthesis to the ecological competence of fluorescent pseudomonads in soil habitats. Applied and Environmental Microbiology 58:2616-2624

McBain AJ (2009) Chapter 4: In vitro biofilm models: an overview. Advances in Applied Microbiology 69:99-132

Mentel M, Ahuja EG, Mavrodi DV, Breinbauer R, Thomashow LS, Blankenfeldt W (2009) Of two make one: the biosynthesis of phenazines. ChemBioChem 10:2295-2304

Murray TS, Egan M, Kazmierczak BI (2007) Pseudomonas aeruginosa chronic colonization in cystic fibrosis patients. Current Opinion in Pediatrics 19:83-88

Nakaike S, Yamagishi T, Nanaumi K, Otomo S, Tsukagoshi S (2005) Cell-killing activity and kinetic analysis of a novel antitumor 
compound NC-190, a Benzo $[\alpha]$ phenazine derivative. Cancer Science 83:402-409

O'May CY, Sanderson K, Roddam LF, Kirov SM, Reid DW (2009) Iron-binding compounds impair Pseudomonas aeruginosa biofilm formation, especially under anaerobic conditions. J Med Microbiol 58:765-773

Parsons JF, Greenhagen BT, Shi K, Calabrese K, Robinson H, Ladner JE (2007) Structural and functional analysis of the pyocyanin biosynthetic protein PhzM from Pseudomonas aeruginosa. Biochemistry 46:1821-1828

Pham TH, Boon N, De Maeyer K, Hofte M, Rabaey K, Verstraete W (2008) Use of Pseudomonas species producing phenazine-based metabolites in the anodes of microbial fuel cells to improve electricity generation. Applied Microbiology and Biotechnology 80:985-993

Pierson LS III, Lam S, Gaffney T, Gong FC (1995) Molecular analysis of genes encoding phenazine biosynthesis in the biological control bacterium Pseudomonas aureofaciens 30-84. FEMS Microbiology Letters 134:299-307

Pierson LS III, Pierson EA (1996) Phenazine antibiotic production by the biological control bacterium Pseudomonas aureofaciens: role in ecology and disease suppression. FEMS Microbiology Letters 136:101-108

Pierson LS, Thomashow LS (1992) Cloning and heterologous expression of the phenazine biosynthetic locus from Pseudomonas aureofaciens 30-84. Molecular Plant-Microbe Interactions 5:330-339

Pinto JE, Dyer WE, Weller SC, Herrmann KM (1988) Glyphosate Induces 3-Deoxy-D-arabino-Heptulosonate 7-Phosphate Synthase in Potato (Solanum tuberosum L) Cells Grown in Suspension Culture. Plant Physiology 87:891-893

Price-Whelan A, Dietrich LE, Newman DK (2006) Rethinking 'secondary' metabolism: physiological roles for phenazine antibiotics. Nat Chem Biol 2:71-78

Price-Whelan A, Dietrich LE, Newman DK (2007) Pyocyanin alters redox homeostasis and carbon flux through central metabolic pathways in Pseudomonas aeruginosa PA14. J Bacteriol 189:6372-6381

Rabaey K, Boon N, Hofte M, Verstraete W (2005) Microbial phenazine production enhances electron transfer in biofuel cells. Environmental Science and Technology 39:3401-3408

Rada B, Leto TL (2009) Redox warfare between airway epithelial cells and Pseudomonas: dual oxidase versus pyocyanin. Immunologic Research 43:198-209

Ran H, Hassett DJ, Lau GW (2003) Human targets of Pseudomonas aeruginosa pyocyanin. Proceedings of the National Academy of Sciences of the United States of America 100: $14315-14320$

Ryazanova OA, Voloshin IM, Makitruk VL, Zozulya VN, Karachevtsev VA (2007) pH-induced changes in electronic absorption and fluorescence spectra of phenazine derivatives. Spectrochim Acta A Mol Biomol Spectrosc 66:849-859

Sanderson DG, Gross EL, Seibert M (1987) A photosynthetic photoelectrochemical cell using phenazine methosulfate and phenazine ethosulfate as electron acceptors. Applied Biochemistry and Biotechnology 14:1-12

Sappal DS, McClendon AK, Fleming JA, Thoroddsen V, Connolly K, Reimer C, Blackman RK, Bulawa CE, Osheroff N, Charlton P, Rudolph-Owen LA (2004) Biological characterization of
MLN944: a potent DNA binding agent. Mol Cancer Ther 3:4758

Schoonbeek HJ, Raaijmakers JM, De Waard MA (2002) Fungal ABC transporters and microbial interactions in natural environments. Molecular Plant-Microbe Interactions 15:1165-1172

Sio CF, Otten LG, Cool RH, Diggle SP, Braun PG, Bos R, Daykin M, Cámara M, Williams P, Quax WJ (2006) Quorum quenching by an $\mathrm{N}$-acyl-homoserine lactone acylase from Pseudomonas aeruginosa PA01. Infection and Immunity 74:1673-1682

Sorensen RU, Klinger JD, Cash HA, Chase PA, Dearborn DG (1983) In vitro inhibition of lymphocyte proliferation by Pseudomonas aeruginosa phenazine pigments. Infection and Immunity 41:321-330

Stewart-Tull DES, Armstrong A (1971) The effect of 1hydroxyphenazine and pyocyanine from Pseudomonas aeruginosa on mammalian cell respiration. J Med Microbiol 5:67-73

Tambong JT, Hofte M (2001) Phenazines are involved in biocontrol of Pythium myriotylum on cocoyam by Pseudomonas aeruginosa PNA1. European Journal of Plant Pathology 107:511-521

Thomashow LS, Weller DM (1988) Role of phenazine antibiotic from Pseudomonas fluorescens in biological control of Gaumannomyces graminis var tritici. J Bacteriol 170:3499-3508

Toohey JI, Nelson CD, Krotkov G (1965) Toxicity of phenazine carboxylic acids to some bacteria, algae, higher plants and animals. Canadian Journal of Botany 43:1151-1155

Torres CI, Marcus AK, Lee HS, Parameswaran P, Krajmalnik-Brown R, Rittmann BE (2010) A kinetic perspective on extracellular electron transfer by anode-respiring bacteria. FEMS Microbiology Reviews 34:3-17

Turner JM, Messenger AJ (1986) Occurrence, biochemistry and physiology of phenazine pigment production. Advances in Microbial Physiology 27:211-275

Van Wees SC, Van der Ent S, Pieterse CM (2008) Plant immune responses triggered by beneficial microbes. Current Opinion in Plant Biology 11:443-448

Verhagen BW, Glazebrook J, Zhu T, Chang HS, van Loon LC, Pieterse CM (2004) The transcriptome of rhizobacteria-induced systemic resistance in Arabidopsis. Molecular Plant-Microbe Interactions 17:895-908

Wang Y, Newman DK (2008) Redox reactions of phenazine antibiotics with ferric (hydr)oxides and molecular oxygen. Environmental Science and Technology 42:2386-2389

Wang Y, Kern SE, Newman DK (2009) Endogenous phenazine "antibiotics" promote anaerobic survival of Pseudomonas aeruginosa via extracellular electron transfer. J Bacteriol 192:365-369

Webby CJ, Baker HM, Lott JS, Baker EN, Parker EJ (2005) The structure of 3-deoxy-D-arabino-heptulosonate 7-phosphate synthase from Mycobacterium tuberculosis reveals a common catalytic scaffold and ancestry for type I and type II enzymes. J Mol Biol 354:927-939

Williams P, Cámara M (2009) Quorum sensing and environmental adaptation in Pseudomonas aeruginosa: a tale of regulatory networks and multifunctional signal molecules. Curr Opin Microbiol 12:182-191

Wink M (2003) Evolution of secondary metabolites from an ecological and molecular phylogenetic perspective. Phytochemistry $64: 3-19$

Winstanley C, Fothergill JL (2009) The role of quorum sensing in chronic cystic fibrosis Pseudomons aeruginosa infections. FEMS Microbiology Letters 290:1-9 